\title{
Heterogeneidad de los efectos de la crisis española en los mercados laborales urbanos
}

Pilar Murias. Universidad de Santiago de Compostela, Santiago de Compostela, España.

Fidel Martínez-Roget. Universidad de Santiago de Compostela, Santiago de Compostela, Espańa.

RESUMEN | Este artículo estudia la heterogeneidad espacial en las consecuencias de la crisis económica de España, al analizar su impacto en diferentes mercados laborales urbanos. Utilizando datos relacionados con los municipios urbanos más grandes de España, se identifican cuatro grupos en función del impacto de la crisis en las tasas de desempleo. Los resultados muestran una importante división norte/sureste en la evolución de los mercados laborales locales en Espańa durante el periodo 2006-2013. Los factores clave de la resiliencia en los mercados laborales urbanos fueron el nivel de estudios de la población, la estructura industrial y, en menor medida, el grado de urbanización.

PALABRAS ClaVe | crisis económica, economía urbana, mercado de trabajo.

ABSTRACT | In this paper, spatial heterogeneity as a consequence of Spain's economic crisis is discussed, and its impact across different urban labour market is analyzed. Using data relating to Spain's largest urban municipalities, we identify four categories of local responses for the rise in unemployment. The results show an important north/south-east divide in the evolution of local labour markets in Spain during the period 2006-2013. The key determinants of resilience in the urban labour markets were the population's skills, the industrial structure and, albeit to a lesser extent, the degree of urbanisation.

KEYWORDS | economic crisis, urban economy, labour market. 


\section{Introducción}

En los últimos años, el interés en el campo del desarrollo regional ha transitado desde la noción de crecimiento a la de resiliencia (Clark, Huang \& Walsh, 2010). A pesar de la complejidad de este último término (Adger, 2000; Davies, 2011; Martin, 2012; Martin, Sunley, Gardiner \& Tyler, 2016), de sus inherentes contradicciones (Dubé \& Polèse, 2016, p. 626; Han \& Goetz, 2015, p. 133; Pike, Dawley \& Tomaney, 2010, p. 63) y de su naturaleza "borrosa", en el sentido que le da Markusen (1999), la mayoría de los autores reconocen su utilidad para las economías regionales y exhortan a un mayor desarrollo teórico y empírico del concepto (Pendall, Foster \& Cowell, 2010). La idea de resiliencia ofrece una alternativa para capturar "la capacidad diferencial y desigual de los lugares para reaccionar, responder y hacer frente a cambios inciertos, volátiles y rápidos" (Pike et al., 2010). Su irrupción supone en gran medida un cambio de paradigma, porque cuestiona los enfoques tradicionales que priorizan la consecución del éxito en un momento concreto del tiempo, en lugar del éxito a lo largo del tiempo (Chapple \& Lester, 2007). Impulsa la superación de estrategias centradas únicamente en el crecimiento y la competitividad (Bristow, 2010), para pasar a considerar perspectivas más amplias, que enfatizan la resistencia de las regiones ante shocks diversos y su capacidad de adaptación a nuevas características del entorno (Clark et al., 2010; Pike et al., 2010).

El cuestionamiento del crecimiento económico y de la competitividad como catalizadores de un mayor desarrollo social y económico responde a muchas causas (Christopherson, Michie \& Tyler, 2010), pero sin duda la reciente y severa crisis mundial ha acelerado la búsqueda de nuevos conceptos que aborden mejor el desempeño económico de las regiones (Hudson, 2010). Esta crisis ha golpeado de un modo extraordinario al entorno de la Unión Europea (UE), que ha sufrido el revés económico más profundo desde la década de 1930 y del que muchos países aún se están recuperando (Comisión Europea, 2009; Cuadrado-Roura \& Maroto, 2016). Una de las economías europeas que más ha sufrido sus efectos es la española. Mientras otros países europeos han iniciado su recuperación a partir de 2010, en España la crisis fue más duradera y no fue sino en el año 2014 que el PIB español empezó a crecer de nuevo (Eurostat, 2018). Factores específicos relacionados con la estructura de la economía y el nivel de endeudamiento hicieron a España más vulnerable (Cuadrado-Roura \& Maroto, 2016). Las cuentas públicas, con un superávit próximo al 2\% del PIB en el año 2007, registraron entre 2009 y 2012 déficits por encima del 9\% del piв (Eurostat, 2018). Tras su deterioro está el desplome de los ingresos públicos por la crisis general —y la inmobiliaria en particular-y un incremento de las partidas de prestación por desempleo. ${ }^{1}$

Precisamente el desempleo fue y es el dato más preocupante para el país, por sus graves consecuencias económicas y sociales. En el año 2013 llegó a alcanzar el $26,1 \%$ de la población activa y en promedio se mantuvo en $20,5 \%$ entre 2008

Para un mayor detalle, pueden consultarse los Presupuestos Generales del Estado de Espańa de los ejercicios 2008-2014, accesibles en http://www.sepg.pap.hacienda.gob.es/sitios/sepg/es-ES/ Presupuestos/PresupuestosEjerciciosAnteriores/Paginas/PresupuestosEjerciciosAnteriores.aspx 
y 2017, más del doble que la media para el conjunto de la ue (Eurostat, 2018). Este dato agregado esconde fuertes diferencias territoriales apreciables claramente a nivel local, ya que los mercados de trabajo tienen generalmente dimensiones subregionales, pero también porque la resiliencia de los territorios tiene en gran medida una base local (Bristow, 2010). De hecho, algunos autores consideran que la resiliencia regional se ve influida considerablemente por las economías locales, eso sí, de carácter urbano (Capello, Caragliu \& Fratesi, 2015).

Precisamente la relación entre resiliencia y grado de urbanización de un territorio ha sido una de las líneas de investigación abierta en los últimos años, heredando la tradición de la literatura que vincula crecimiento y grado de urbanización (Duranton \& Puga, 2014; Glaeser \& Kahn, 2004). Los trabajos, en aumento, son aun relativamente escasos. No existe tampoco consenso en las conclusiones, aunque parecen más los autores que consideran que la resiliencia no está necesariamente ligada a mayores niveles de urbanización y que, al menos en el corto plazo, el impacto de la última crisis económica ha sido mayor en las ciudades, especialmente en términos de desempleo. Se hace necesario, por lo tanto, analizar nuevas evidencias para profundizar en la relación entre la resiliencia y "lo urbano", así como examinar los factores que caracterizan las ciudades más y menos resistentes.

En esta línea se enfoca el presente artículo, que analiza la incidencia del desempleo a nivel urbano en España después de la crisis de 2008 y, por tanto, la capacidad de los mercados de trabajo urbanos para resistir ante dicha crisis. El trabajo puede contribuir al debate abierto a través de la comparación entre el impacto sufrido por los municipios urbanos y el experimentado por las regiones en las que se insertan, o las áreas no urbanas de las mismas. Además, permite analizar si la crisis ha contribuido o no a reducir las diferencias territoriales. El artículo también contribuye a la literatura analizando las características de los municipios urbanos con mejor y peor comportamiento, identificando actores económicos, sociales y demográficos que pueden estar detrás de la resiliencia a corto plazo de los mercados de trabajo urbanos en España.

El texto está estructurado de la siguiente manera: tras esta introducción, la segunda sección hace una breve revisión de los trabajos que han analizado empíricamente la relación entre resiliencia y grado de urbanización. En el tercer apartado se analiza el impacto de la crisis en los grandes municipios urbanos españoles. A continuación se presentan diversas cuestiones metodológicas, para centrarse en la identificación de factores que caracterizan a los municipios urbanos más y menos resistentes a la crisis. Finalmente se señalan las conclusiones, junto con las limitaciones del estudio y futuras líneas de ampliación del mismo.

\section{Resiliencia y grado de urbanización}

La introducción del concepto de resiliencia en la economía regional se basa en la ampliación del enfoque de resiliencia de ecosistemas (Holling, 1973). Pese a algunos precedentes importantes, puede decirse que los primeros marcos teóricos para el estudio de la resiliencia regional (Chapple \& Lester, 2007; Hill, Wial \& Wolman, 2008; Hill et al., 2012; Pendall, Foster $\&$ Cowell, 2007) surgieron a partir 
de 2006 con la iniciativa Building Resilient Regions de la MacArthur Foundation. Aun así, el acontecimiento que impulsó definitivamente el concepto dentro de la economía regional fue la crisis económica internacional conocida como la Gran Recesión y surgida a partir de la crisis financiera de 2007. La Gran Recesión supuso una motivación decisiva para la utilización del concepto de resiliencia en el análisis de los shocks económicos y sus heterogéneos efectos sobre los territorios (Bristow, 2010; Courvisanos, Jain \& Mardaneh, 2016; Martin, 2012).

Uno de los marcos teóricos que mejor describen la complejidad del concepto y con mayor aceptación en la comunidad científica es el de Martin (2012). El autor identifica cuatro dimensiones de resiliencia, revisando las implicaciones de estudiar cada una de ellas: resistencia, recuperación, reorientación y renovación. La primera dimensión, resistencia, en la que se centra este trabajo, evalúa la capacidad de un territorio para mantenerse en su posición inicial previa al impacto del shock. Por lo tanto, la resiliencia en su dimensión de resistencia mide la vulnerabilidad de una economía ante un shock, es decir, la profundidad del impacto sufrido por la misma. La capacidad de resistencia es la dimensión de la resiliencia más fácil de abordar, en la medida en que se puede observar en un plazo de tiempo más corto, aunque este análisis es necesariamente parcial, porque una región resistente en el corto plazo puede ser poco adaptable en el largo plazo. En general, una región aparentemente exitosa cuando se considera un periodo específico de tiempo, puede constituir una realidad opuesta ante una escala temporal más amplia, o viceversa (Pendall et al., 2010, p. 80). Por ejemplo, Christopherson et al. (2010, p. 6) señalan cómo entre las áreas que mejor han sorteado la crisis en Estados Unidos se encuentran algunas antiguas zonas industriales en declive que no resultaron atractivas durante la burbuja inmobiliaria y que ahora no pagan los efectos negativos que ella tuvo. Las que parecían áreas claramente ganadoras, parecen no haberlo sido tanto a la luz de los acontecimientos actuales.

Desde la perspectiva espacial, el estudio de la resistencia a la crisis o del impacto de la misma a nivel local es defendido por varios autores que consideran que la capacidad de respuesta ante una crisis surge a nivel local, y también que la acción local es indispensable para afrontar las consecuencias de la crisis (Bailey \& Chapain, 2011; Bristow, 2010; Champion \& Townsend, 2011; Hudson, 2010; Lee, Morris $\&$ Jones, 2009; Shaw, 2012). En cualquier caso, nuevamente, Pendall et al. (2010, p. 79) subrayan que la consideración de distintas fronteras regionales puede alterar los resultados en cuanto a resiliencia (Modifiable Areal Unit Problem: Fotheringham \& Wong, 1991; Openshaw, 1984). Tras tal enfoque está el hecho de que un análisis de la resiliencia a nivel regional esconderá con frecuencia importantes diferencias intrarregionales. Por ejemplo, Hill et al. (2012) muestran que, aunque la región de Detroit puede ser considerada como resiliente ante los shocks experimentados a partir del año 2000, la ciudad no podría calificarse como tal.

Siguiendo esta corriente, en los últimos años diversos trabajos empíricos han analizado el impacto diferencial de la crisis reciente sobre países, regiones y territorios. Entre ellos, algunos se han centrado en las economías urbanas, en la perspectiva de analizar si el impacto de la crisis ha sido mayor o menor en estas áreas en comparación con el resto de la región. Estos estudios han tomado a menudo 
el mercado laboral como referencia y, como indicador, la tasa de desempleo. A continuación, se revisan algunos de los más relevantes.

Brakman, Garretsen y van Marrewijk (2015) analizaron la evolución de regiones de la Unidad Europea y encontraron que las más urbanas experimentaron un mayor incremento del desempleo. Resultados similares fueron obtenidos para las regiones europeas de la Organización para la Cooperación y el Desarrollo Económicos (ocDE) por Dijkstra, Garcilazo y McCann (2015). A su vez, Giannakis y Bruggeman (2017) encontraron que el desempleo en Grecia aumentó más en las regiones urbanas que en las rurales. Palaskas, Psycharis, Rovolis y Stoforos (2015) obtienen que, entre los municipios urbanos griegos, precisamente los más urbanizados han sufrido más el incremento del desempleo. Los autores hablan incluso de una "crisis de urbanización" en Europa como consecuencia de que los efectos de la crisis económica han sido más evidentes en las ciudades, apoyándose en Cohen (2011) у URBACt (2010), mientras que Cuadrado-Roura y Maroto (2016) hablan de la "dimensión urbana" de la crisis. Por su parte, Parkinson et al. (2012) afirman que las ciudades europeas "de segundo orden" fueron severamente impactadas por la crisis. Estos resultados coinciden con los de Champion y Townsend (2013) y Townsend y Champion (2014), que concluyen que este tipo de ciudades sufrió más el incremento de desempleo que el resto del Reino Unido.

Sin embargo, estos últimos autores sostienen que el entorno de la gran ciudad de Londres resistió mejor la crisis, lo que concuerda con la reflexión de Mrinska y Smetkowski (2013, p. 13), según la cual las capitales de la mayor parte de los países europeos aguantaron relativamente bien. Otros autores tienen una visión aún más positiva de la resiliencia de las áreas urbanas, como Capello et al. (2015) o Fratesi y Perucca (2018), quienes afirman que las áreas más resilientes son aquellas más urbanas, matizando que reaccionaron con mayor rapidez que las menos urbanas, pese a haber sido inicialmente las más afectadas.

Desde el punto de vista teórico, existen argumentos a favor y en contra de una relación positiva entre resiliencia y nivel de urbanización. Cabe esperar una mayor resiliencia de las ciudades antes la crisis económica si atendemos a la diversificación del tejido productivo y a las economías de aglomeración que caracterizan estas áreas (Capello et al., 2015; Duranton \& Puga, 2014; Glaeser \& Kahn, 2004; McCann, 2013; Polèse, 2010). Sin embargo, otros autores consideran que los entornos urbanos fueron más vulnerables a corto plazo respecto a la crisis de 2008 (Capello et al., 2015; Dijkstra et al., 2015; Palaskas et al., 2015, p. 978; Polèse, 2010), especialmente por los sectores en los que ella se originó: finanzas, construcción, bienes raíces... (Harvey, 2012). En concreto, Dijkstra et al. (2015) apuntan, además, que las áreas urbanas están más conectadas con los mercados mundiales, por lo que sufrieron especialmente la caída en los flujos comerciales y financieros internacionales. Señalan, además, que los argumentos tradicionales sobre economías de aglomeración funcionan mejor en condiciones de crecimiento que en las de crisis económica. Por su parte, Brakman et al. (2015) apuntan que resulta difícil separar los efectos del grado de urbanización de aquellos de la composición sectorial del tejido económico, un factor que se considera fundamental para el crecimiento y el empleo en un área geográfica, y que puede serlo también para la resiliencia. Los 
autores hablan incluso de "dos caras de la misma moneda". En su estudio para más de 200 regiones europeas, encuentran que ambos aspectos afectan individual y conjuntamente a la resiliencia de un territorio. Son las áreas intermedias (commuting areas) cercanas a las grandes ciudades y aquellas con mayor peso de sectores de alta y media tecnología las que resistieron mejor el impacto inicial de la crisis. Los autores seńalan que las áreas urbanas en torno a las grandes ciudades presentan con frecuencia fuerza de trabajo con mayor movilidad y alto nivel formativo, y que además resultan atractivas para la localización de ciertos tipos de empresas.

En el caso concreto de los entornos urbanos españoles, Caravaca, GonzálezRomero y López (2017) analizaron los municipios de más de 20.000 habitantes, concluyendo que la mayoría ha tenido una evolución del desempleo peor o, en el mejor de los casos, similar a la media nacional, y solo unos pocos han tenido mejores resultados. Los autores relacionan este último comportamiento con la composición sectorial; en concreto, con el mayor peso de actividades intensivas en conocimiento. Tomando como referencia este trabajo, en los siguientes apartados se analizará el impacto en los grandes municipios urbanos (mayores de 50.000 habitantes) y se relacionará este impacto con una serie de factores económicos, demográficos y sociales de los mismos.

\section{La resiliencia a corto plazo de los mercados laborales en los grandes municipios urbanos españoles}

\section{Crecimiento económico y crisis en España}

La evolución reciente del desempleo en España ha atraído la atención de los comentaristas económicos, que en un corto periodo han convertido al país en un ejemplo, aunque por razones opuestas (de la Dehesa, 2003; Sacht, 2015; Vińuela, Rubiera \& Cueto, 2012). La economía española mostró hasta 2008 un comportamiento dinámico, con una creación de empleo entre las más altas de la UE. Considerando el periodo 1990 a 2008, la tasa media anual de crecimiento del país rondó el 3\% (Maddison, 2009), a pesar de la desaceleración en el inicio de ambas décadas. Aunque tradicionalmente la economía espańola presentaba niveles de desempleo comparativamente altos para el contexto europeo, estos se habían reducido considerablemente durante el periodo de crecimiento. En el ańo 2007 la tasa de desempleo en España se situaba en 8,3\%, siete décimas por encima de la media de la Eurozona (7,6\%), cuando en 1993 , con un $20,1 \%$, doblaba dicha media $(10,2 \%)$ (Eurostat, 2018). La reducción del desempleo, impulsada fundamentalmente por el auge del sector de la construcción, parecía incluso más meritoria si se tiene en cuenta los importantes flujos de población inmigrante recibida (Muñoz de Bustillo \& Antón, 2010). En este panorama aparentemente exitoso había, sin embargo, dos importantes matices negativos. Por un lado, el mal comportamiento de la productividad, prácticamente estancada, y que hacía que el crecimiento económico dependiese exclusivamente del factor trabajo empleado. Esta circunstancia justifica que se hablase de un país que crecía por "transpiración” y no por "inspiración” (van Ark, O’Mahony \& Ypma, 2007). Por otra parte, se advertía la consolidación de un 
mercado laboral segmentado, con importantes diferencias entre trabajadores indefinidos y temporales en términos de condiciones de trabajo y perspectivas en sus carreras profesionales (Europa Publications, 2009; Muńoz de Bustillo, 2007).

Los aspectos negativos del crecimiento espańol se hicieron especialmente patentes cuando dicho proceso se convirtió en desaceleración primero, y recesión después, a lo largo del año 2008. La crisis económica afectaba a la mayoría de los países desarrollados y hubo claros factores externos, pero una vez más los desequilibrios internos pasaron factura a la economía española. La burbuja inmobiliaria, la excesiva dependencia de la financiación exterior, la dependencia de la energía importada y de sectores tan sensibles a los cambios en la renta como el turismo, causaron una importante reducción de la demanda, crecimiento del déficit público y, sobre todo, un inexorable incremento del desempleo. En este sentido, España concentró la mayor parte del deterioro del mercado laboral de la Zona Euro (el 60\% de los empleos destruidos de la Zona eran espańoles). La tasa de desempleo, que en términos medios para Espańa era del 8,3\% en 2007, pasó al 11\% en 2008 y al $18 \%$ en 2009, para alcanzar la cota máxima en el año 2013, con un valor superior al 26\% (Instituto Nacional de Estadística, 2017b). En ese año, dicha tasa superaba en más de 15 puntos la media de la UE-28 y solo Grecia registraba una tasa más elevada (Eurostat, 2018).

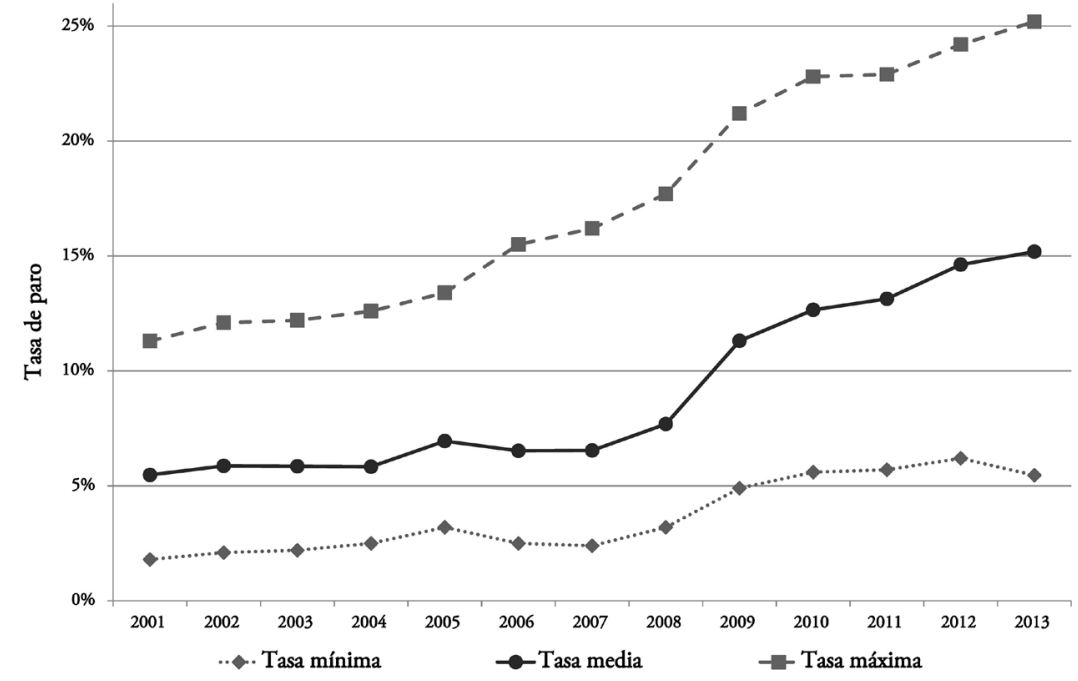

Figura I | Evolución de las tasas de desempleo en municipios con más de 50.000 habitantes

FUENTE ELABORACiÓN PROPIA BASADA EN PARO REGISTRADO EN PORCENTAJE DE LA POBLACIÓN POTENCIALMENTE ACTIVA (LA CAIXA, 20I3) 
Los costes de la crisis fueron especialmente graves a nivel local. El estallido de la burbuja inmobiliaria tuvo una específica repercusión en las finanzas locales, principalmente a través de la reducción drástica de los ingresos "extraordinarios", que dependían del flujo de viviendas construidas y no de su stock (Solé-Ollé \& Viladecans-Marsal, 2011). Esta merma de ingresos, junto con las restricciones presupuestarias derivadas de la política de austeridad adoptada en el marco de la UE, dejó prácticamente sin margen de maniobra a las instituciones locales para impulsar iniciativas que paliaran los efectos de la crisis sobre el mercado laboral. Los mercados de trabajo urbanos sufrieron de manera desigual las consecuencias de la crisis (Caravaca et al., 2017). Las diferencias en las tasas de desempleo municipales (Figura 1), que habían aumentado desde comienzos de siglo, persistieron en los ańos de crisis económica. En definitiva, la probabilidad de estar desempleado parece variar considerablemente entre un municipio y otro.

El impacto de la crisis en el desempleo de los grandes municipios urbanos españoles El punto de partida del análisis empírico es el estudio de la resiliencia de los mercados laborales frente a la crisis de 2008 en los 145 municipios urbanos españoles de más de 50.000 habitantes. Para ello, se compararon las tasas de desempleo de cada municipio entre 2006 y 2013 (antes del estallido de la crisis y en su punto máximo). Para analizar el impacto diferencial de la crisis en los 145 municipios, se ha optado por clasificarlos distinguiendo primero en función de que el aumento del desempleo sea inferior o superior a la media, y discriminando posteriormente los comportamientos extremos en ambos casos (Méndez, Abad \& Echaves, 2015). De este modo resultan los siguientes grupos:

- Grupo 1: municipios urbanos en los que el crecimiento de la tasa de desempleo durante el periodo fue inferior a la media en un valor superior a una desviación típica. Son aquellos en los que la crisis ha tenido un menor impacto en términos de crecimiento del desempleo.

- Grupo 2: municipios urbanos en los que el crecimiento de la tasa de desempleo durante el periodo es inferior a la media, pero en un valor inferior a una desviación típica.

- Grupo 3: municipios urbanos en los que el crecimiento de la tasa de desempleo en el periodo ha sido superior a la media, pero en un valor inferior a una desviación típica.

- Grupo 4: municipios urbanos que han experimentado durante el periodo un crecimiento de la tasa de desempleo superior a la media y además en un valor superior a una desviación típica. Son aquellos en los que la crisis ha tenido un impacto mayor en la evolución del desempleo.

Como se recoge en la Tabla 1, de los 145 municipios urbanos analizados, 12 quedarían incluidos en el grupo 1, 46 en el grupo 2, 59 en el grupo 3 y 28 en el grupo 4. En la tabla se puede ver, además, una relación detallada de los municipios incluidos en cada grupo y su distribución regional. 


\begin{tabular}{|c|c|c|c|c|c|c|c|c|c|}
\hline 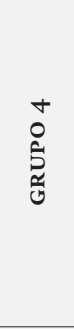 & 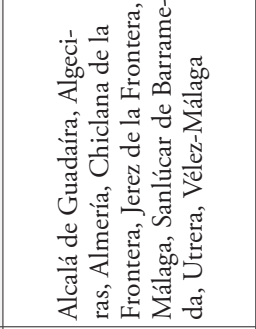 & & & & 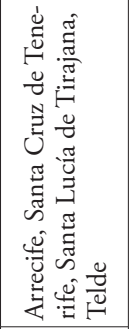 & & & 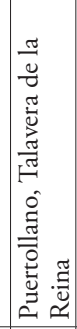 & \\
\hline 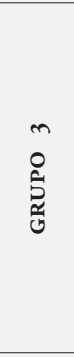 & 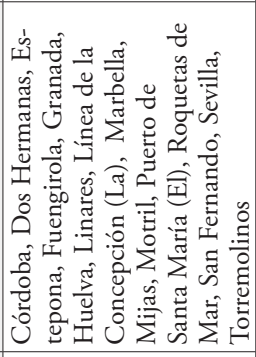 & 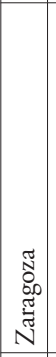 & & & 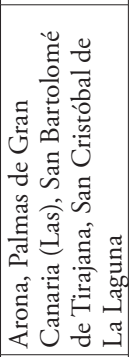 & . & 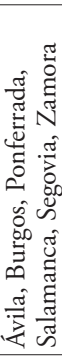 & 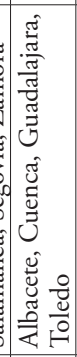 & 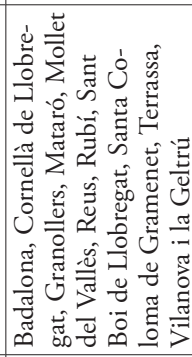 \\
\hline 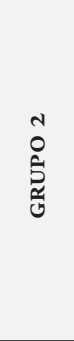 & 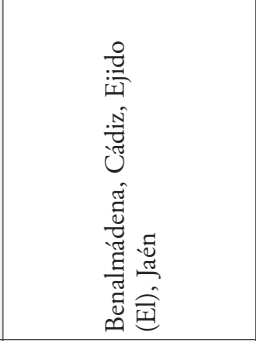 & 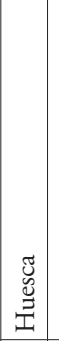 & 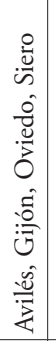 & 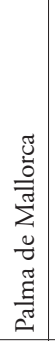 & & 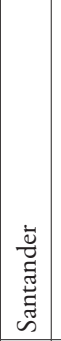 & 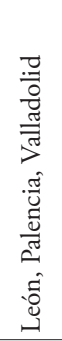 & 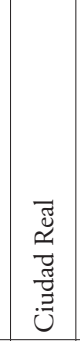 & 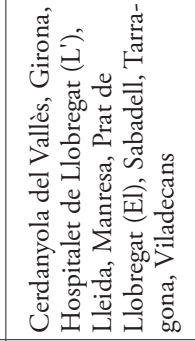 \\
\hline 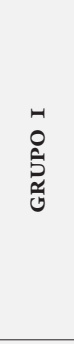 & & & & $\frac{3 \pi}{\frac{\pi}{n}}$ & & & & & 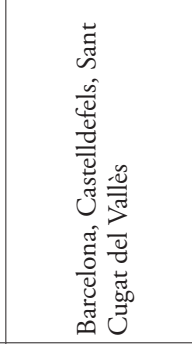 \\
\hline 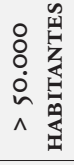 & సे & $\sim$ & $\forall$ & $\sim$ & $\infty$ & $N$ & $a$ & $n$ & $\ddot{\sim}$ \\
\hline 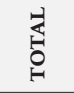 & $\vec{\wedge}$ & $\vec{n}$ & $\stackrel{\infty}{\sim}$ & $\hat{\sigma}$ & $\infty$ & Õ & $\underset{\stackrel{\infty}{+}}{\stackrel{\sim}{N}}$ & $\frac{\approx}{\sigma}$ & ثै \\
\hline 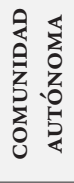 & 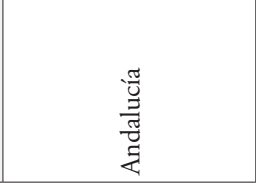 & 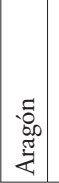 & 䋨 & 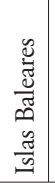 & 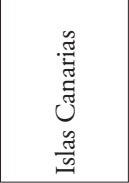 & 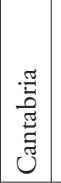 & 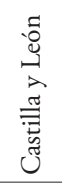 & 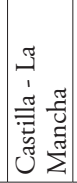 & 莺 \\
\hline
\end{tabular}




\begin{tabular}{|c|c|c|c|c|c|c|c|c|c|c|c|c|}
\hline 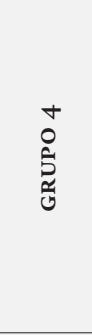 & 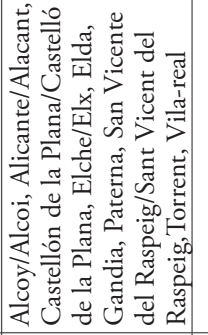 & & & & $\begin{array}{l}\frac{\pi}{\pi} \\
\approx\end{array}$ & 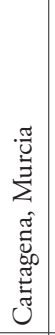 & & & & & $\stackrel{\sim}{\sim}$ & 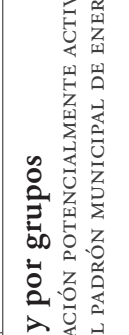 \\
\hline $\begin{array}{l}n \\
0 \\
5 \\
0 \\
0 \\
0\end{array}$ & 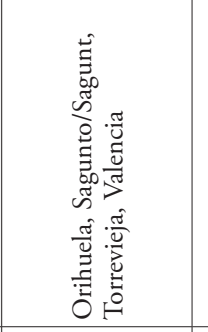 & 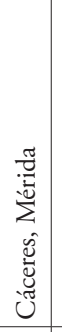 & & 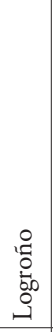 & 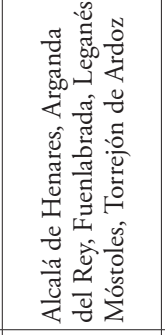 & 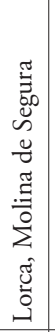 & & 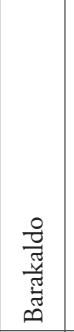 & & & nิ & 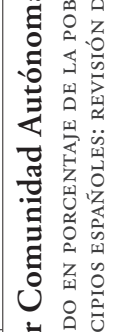 \\
\hline 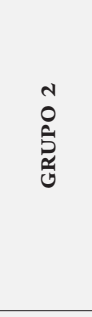 & 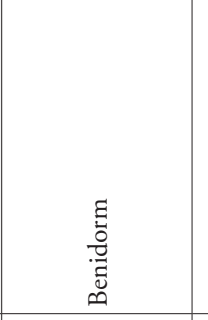 & 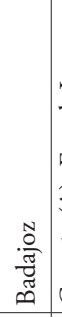 & 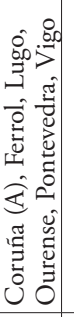 & & 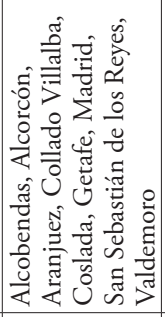 & & 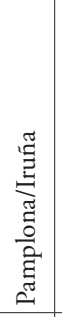 & 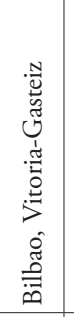 & Uूँ & 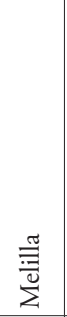 & ¿্t & 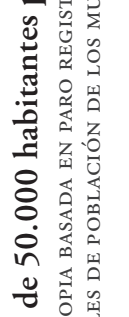 \\
\hline 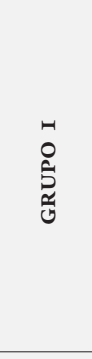 & & & 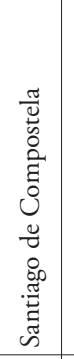 & & 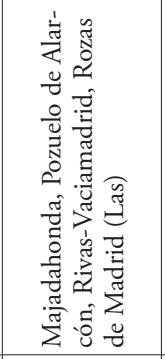 & & & 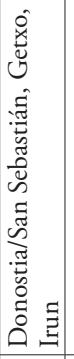 & & & $\simeq$ & 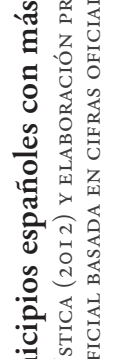 \\
\hline 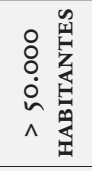 & 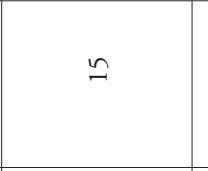 & $m$ & $\wedge$ & - & 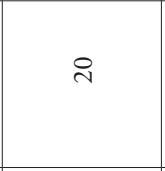 & $\nabla$ & - & 0 & - & - & $\stackrel{n}{ \pm}$ & 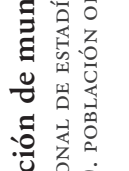 \\
\hline 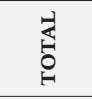 & Iี & $\begin{array}{l}n \\
\infty \\
m\end{array}$ & $\frac{n}{n}$ & 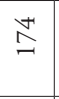 & શ & 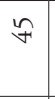 & $\underset{\sim}{\mathbb{N}}$ & $\overrightarrow{\widetilde{\sim}}$ & - & - & $\underset{\infty}{\stackrel{0}{\exists}}$ & 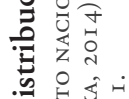 \\
\hline 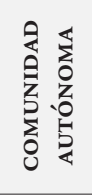 & 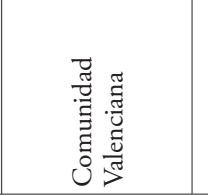 & 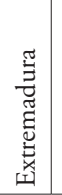 & 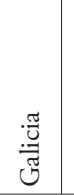 & 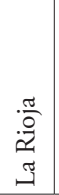 & : & $\stackrel{\vec{s}}{\Sigma}$ & 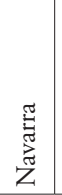 & 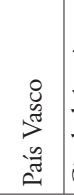 & 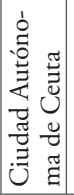 & 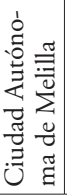 & 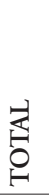 & 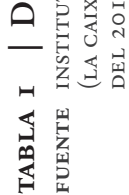 \\
\hline
\end{tabular}


La composición de los grupos revela diferencias espaciales en la resiliencia en Espańa. Más específicamente, se evidencian importantes contrastes entre norte y sur del país, como puede comprobarse en la Figura 2. Los municipios urbanos que mejor han resistido la crisis se sitúan en las regiones del norte, como Asturias, Baleares, Galicia y Navarra, cuyos grandes municipios urbanos pertenecen a los grupos 1 y 2. El País Vasco también ha soportado la crisis mejor que la media, con cinco de sus seis grandes municipios en esos dos grupos. En cambio, los municipios urbanos más impactados por la crisis pertenecen a regiones del sur (Andalucía, Murcia, Comunidad Valenciana y las Islas Canarias). Prácticamente la totalidad de los municipios de estas comunidades se clasifican en los grupos 3 y 4 . Además, aquellos que sufrieron con mayor intensidad los efectos de la crisis, los del grupo 4, se localizan mayoritariamente en estas regiones.

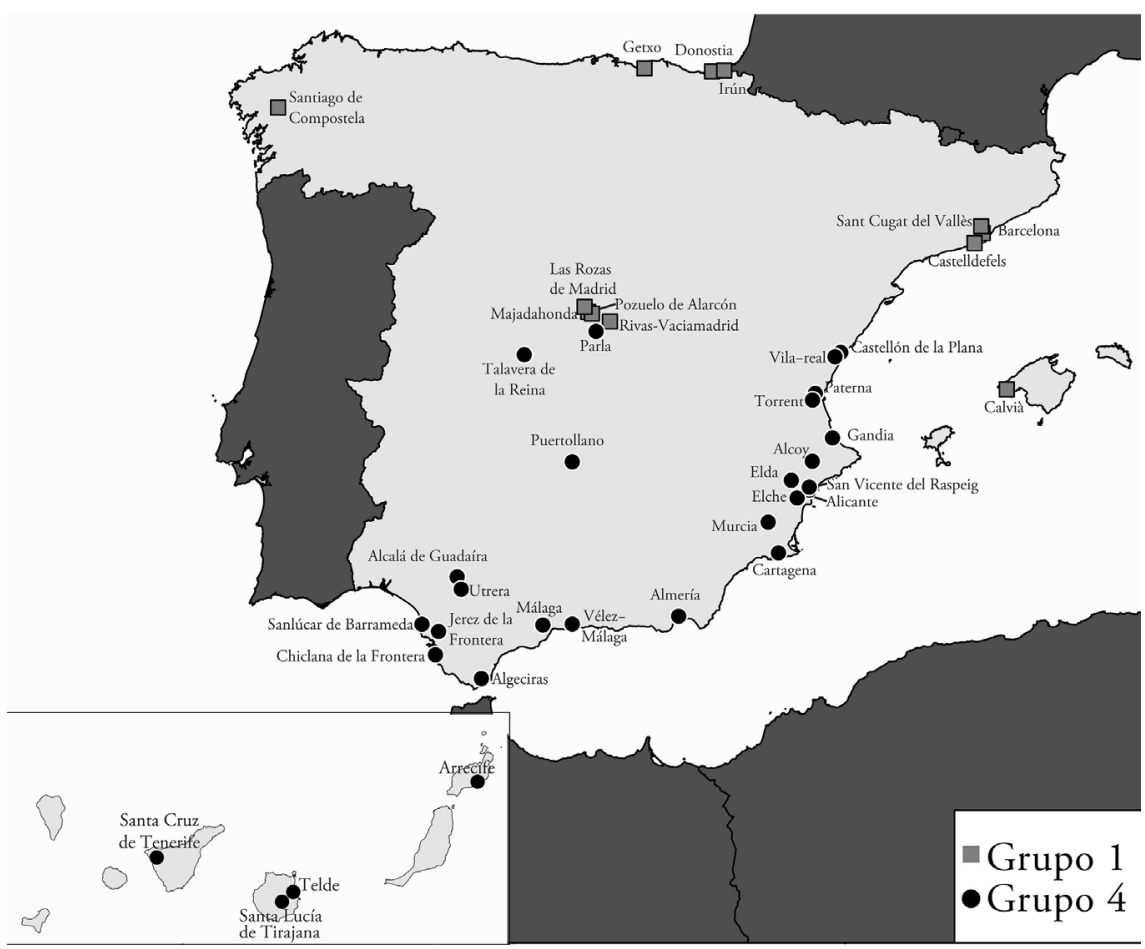

FIGURA 2 Distribución geográfica de los municipios de los grupos 1 y 4 FUENTE ELABORACIÓN PROPIA

Entre los municipios que mejor consiguieron contener el crecimiento del desempleo durante la crisis económica (grupos 1 y 2), algunos, como Calviá o Pozuelo de Alarcón, lo hicieron, además, partiendo de niveles muy bajos de desempleo en el ańo 2006 (2,5\% y 3\%, respectivamente), mientras que otros, como Melilla, Ceuta o Cádiz, partían de niveles más elevados (cuatro veces más que los anteriores) y, por lo tanto, tenían a priori un menor margen de empeoramiento. Sin embargo, otros 
municipios que también partían de niveles elevados de desempleo, como Elda o Alcalá de Guadaíra, no solo no contuvieron su crecimiento, sino que se encuentran en el grupo que más ha crecido en el periodo considerado. La situación de partida tampoco es idéntica entre los municipios que incrementaron su desempleo por encima de la media en el periodo analizado. Por ejemplo, dos municipios clasificados en el grupo 4, Castellón de la Plana y Elda, han registrado un incremento similar en la tasa de paro entre los ańos 2006 y 2013, pero mientras el primero partía de una tasa de paro de 4\%, Elda registraba ya un $12 \%$ el año 2006. Por lo tanto, no se observa un patrón homogéneo dentro de los grupos en función del nivel inicial del desempleo.

Comparando la evolución de la tasa de desempleo entre grandes municipios urbanos y el resto de los municipios de la región, se observa que, en 15 de las 17 regiones españolas, el impacto de la crisis ha sido más grave en los grandes núcleos urbanos (Tabla 2). En el caso de los grandes municipios con tasas de desempleo elevadas al inicio del periodo, su situación relativa con respecto al resto de municipios empeoró como resultado de la crisis. Un ejemplo de esto son La Rioja, Aragón y las Islas Baleares, donde entre los ańos 2006 y 2013, la tasa de desempleo promedio en los municipios de más de 50.000 habitantes aumentó en torno a 1,5 puntos más que en el resto de la región. Resultados similares se observan en Cantabria, Murcia, País Vasco y Castilla y León, aunque en menor medida, con diferencias que oscilan entre 0,5 y 0,4 puntos. Castilla-La Mancha y Madrid son las únicas regiones donde las grandes ciudades obtuvieron mejores resultados que otros municipios, aunque en este último caso, la tasa de desempleo fue solo dos décimas de punto más alta en los grandes centros de población que en el resto de la región.

Si se compara la situación del mercado laboral en el periodo 2006-2013, se observa que la crisis ha contribuido a incrementar las desigualdades regionales, al menos en lo que al contexto urbano se refiere. Fue precisamente en las regiones con mayor nivel de desempleo inicial, como Andalucía, Extremadura o Canarias, donde más creció el desempleo de las grandes áreas urbanas. Por el contrario, en las regiones con una mejor situación de partida en el mercado laboral (Baleares, Cataluña, Madrid, País Vasco o Navarra), el impacto de la crisis sobre el empleo de sus grandes municipios urbanos ha sido menor.

Las excepciones a este patrón se localizan en el comportamiento de los municipios urbanos de cuatro regiones. Por un lado, los municipios de Asturias y Galicia, regiones que también partían de tasas de desempleo superiores a la media, muestran una resiliencia mayor y han acabado con tasas de desempleo por debajo o muy próximas a la media. En cambio, los municipios urbanos de la Comunidad Valenciana y Murcia, comunidades con bajos niveles de desempleo inicial, muestran una baja resiliencia, acabando el periodo con tasas superiores a la media de los municipios analizados. 


\begin{tabular}{|c|c|c|c|c|}
\hline \multirow[t]{2}{*}{ REGIÓN } & \multicolumn{2}{|c|}{$\begin{array}{l}\text { T.D. MEDIA DE LOS MUNICIPIOS } \\
\text { URBANOS POR REGIÓN }\end{array}$} & \multicolumn{2}{|c|}{$\begin{array}{c}\text { DIFERENCIA ENTRE T.D. MEDIA } \\
\text { EN MUNICIPIOS URBANOS Y NO } \\
\text { URBANOS POR REGIÓN }\end{array}$} \\
\hline & 2006 & 2013 & 2006 & 2013 \\
\hline Andalucía & $6,6 \%$ & $13,5 \%$ & 1,5 & 1,6 \\
\hline Aragón & $3,0 \%$ & $9,0 \%$ & 0,6 & 2,0 \\
\hline Canarias & $7,1 \%$ & $14,4 \%$ & 1,0 & 1,3 \\
\hline Cantabria & $4,1 \%$ & $9,9 \%$ & 0,7 & 1,1 \\
\hline Castilla - La Mancha & $4,9 \%$ & $11,7 \%$ & 0,6 & $-0,4$ \\
\hline Castilla y León & $4,7 \%$ & $10,1 \%$ & 0,9 & 1,3 \\
\hline Cataluña & $3,7 \%$ & $8,4 \%$ & 0,5 & 0,5 \\
\hline Comunidad de Madrid & $3,5 \%$ & $8,4 \%$ & 0,3 & 0,0 \\
\hline $\begin{array}{l}\text { Comunidad Foral de } \\
\text { Navarra }\end{array}$ & $3,8 \%$ & $8,4 \%$ & 0,8 & 0,8 \\
\hline Comunidad Valenciana & $4,2 \%$ & $11,5 \%$ & 0,5 & 0,6 \\
\hline Extremadura & $7,1 \%$ & $13,1 \%$ & 0,1 & 0,3 \\
\hline Galicia & $6,1 \%$ & $10,3 \%$ & 0,8 & 0,8 \\
\hline Islas Baleares & $3,1 \%$ & $7,7 \%$ & 0,8 & 2,2 \\
\hline La Rioja & $3,5 \%$ & $9,4 \%$ & 0,6 & 2,1 \\
\hline País Vasco & $3,8 \%$ & $8,4 \%$ & 0,4 & 0,9 \\
\hline Principado de Asturias & $5,0 \%$ & $10,0 \%$ & 0,8 & 1,0 \\
\hline Región de Murcia & $2,8 \%$ & $10,2 \%$ & $-0,5$ & 0,0 \\
\hline
\end{tabular}

\section{TABLA 2 Evolución de las tasas de desempleo (TD) por regiones}

FUENTE ELABORACIÓN PROPIA BASADA EN PARO REGISTRADO EN PORCENTAJE DE LA POBLACIÓN POTENCIALMENTE ACTIVA (LA CAIXA, 20I4)

Los resultados no parecen mostrar una particular relación entre el grado de resistencia de los grandes municipios urbanos y su tamaño poblacional. Aun así, el grupo 1 está formado mayoritariamente por municipios urbanos de tamaño relativamente pequeño (menos de 100.000 habitantes), con las excepciones significativas de un municipio urbano medio, como San Sebastián, y un gran municipio urbano, como Barcelona. Esta regularidad no se observa en ninguno de los restantes grupos. Por ejemplo, en el grupo 4 están grandes municipios como Murcia o Málaga, municipios medianos como Tenerife, Elche o Almería, y varios relativamente pequeños, como Vila-real, Vélez-Málaga o Alcoy.

Tampoco parece que se puedan establecer patrones claros atendiendo a las funciones de las áreas urbanas en las que estos municipios se insertan. En particular, la resiliencia en los grandes municipios urbanos pertenecientes a alguna de las cinco áreas urbanas calificadas como Áreas Europeas Metropolitanas de Crecimiento (MEGA, por sus siglas en inglés: Metropolitan European Growth Areas) ${ }^{2}$ (EsPON, 2003), en España varía considerablemente (Tabla 3). Valencia, Torrent y Paterna, municipios del área urbana de Valencia, están clasificados en los grupos menos resilientes. Algo similar ocurre con los municipios analizados que se integran en el área

2 La iniciativa ESPON (European Spatial Planning Observation Network) clasifica como mega en Espańa, las áreas funcionales urbanas de Madrid, Barcelona, Valencia, Sevilla y Palma de Mallorca. 
funcional urbana de Sevilla. Los resultados de estos municipios integrados en una MEGA son similares a los de otros grandes municipios urbanos de la misma región fuera de la mega. Sin embargo, Calviá y Palma de Mallorca, los dos únicos municipios mayores de 50.000 habitantes del área funcional urbana de Palma de Mallorca, son clasificados en los grupos 1 y 2, respectivamente. El comportamiento de los grandes municipios urbanos de las áreas funcionales de Madrid y Barcelona es más dispar. En el caso de Madrid, hay municipios del área en todos los grupos, aunque en un $63 \%$ se clasificaron en los grupos 1 y 2 . La situación es parecida en el área funcional de Barcelona, que no tiene municipios en el grupo 4 y con el $44 \%$ de los municipios urbanos del área clasificados como resilientes o relativamente resilientes.

\begin{tabular}{|c|c|c|c|c|}
\hline \multirow{2}{*}{ MEGA } & \multicolumn{4}{|c|}{ GRUPOS } \\
\hline & $\mathbf{I}$ & 2 & 3 & 4 \\
\hline \multirow{10}{*}{ Barcelona } & Barcelona & Cerdanyola del Vallès & Badalona & \\
\hline & Castelldefels & $\begin{array}{l}\text { L'Hospitalet de } \\
\text { Llobregat }\end{array}$ & Cornellà de Llobregat & \\
\hline & Sant Cugat del Vallès & El Prat de Llobregat & Granollers & \\
\hline & & Sabadell & Mataró & \\
\hline & & Viladecans & Mollet del Vallès & \\
\hline & & & Rubí & \\
\hline & & & Sant Boi de Llobregat & \\
\hline & & & $\begin{array}{l}\text { Santa Coloma de } \\
\text { Gramenet }\end{array}$ & \\
\hline & & & Terrassa & \\
\hline & & & Vilanova i la Geltrú & \\
\hline \multirow{8}{*}{ Madrid } & Majadahonda & Alcobendas & Alcalá de Henares & \multirow{8}{*}{ Parla } \\
\hline & Pozuelo de Alarcón & Alcorcón & Arganda del Rey & \\
\hline & Rivas-Vaciamadrid & Collado Villalba & Fuenlabrada & \\
\hline & Las Rozas de Madrid & Coslada & Leganés & \\
\hline & & Getafe & Móstoles & \\
\hline & & Madrid & Torrejón de Ardoz & \\
\hline & & $\begin{array}{l}\text { San Sebastián de los } \\
\text { Reyes }\end{array}$ & & \\
\hline & & Valdemoro & & \\
\hline $\begin{array}{l}\text { Palma de } \\
\text { Mallorca }\end{array}$ & Calvià & Palma de Mallorca & & \\
\hline \multirow{2}{*}{ Sevilla } & & & Dos Hermanas & Alcalá de Guadaíra \\
\hline & & & Sevilla & \\
\hline \multirow{2}{*}{ Valencia } & & & \multirow{2}{*}{ Valencia } & Paterna \\
\hline & & & & Torrent \\
\hline
\end{tabular}

TABLA 3 | Distribución por grupos de los municipios de las áreas funcionales MEga en España

FUENTE ELABORACIÓN PROPIA CON BASE EN ESPON (2003) 
Teniendo en cuenta que no se encuentran patrones definidos de comportamiento en relación con estas dos variables, en el apartado siguiente se va a usar un análisis discriminante para registrar las diferencias entre grupos en función de un conjunto de variables económicas, sociales y demográficas.

\section{Características de los municipios más y menos resistentes}

El análisis discriminante, desarrollado a partir de los trabajos de Fisher (1936) y utilizado en el análisis de la resiliencia (por ejemplo, Chapple y Lester, 2010, o Eraydin, 2016), permite la discriminación de grupos en función de un conjunto de variables independientes. La técnica se fundamenta en construir una función discriminante como una combinación lineal de las variables (denominadas variables canónicas) que permita la mayor discriminación posible entre los grupos definidos a priori, de tal manera que para diferenciar entre $n$ grupos se obtienen $n-1$ funciones discriminantes. Para ello se establecen las ponderaciones del valor teórico para cada variable de forma que maximicen la varianza intergrupos frente a la varianza intragrupos.

Para contrastar la significación estadística de las funciones discriminantes, se analiza a través de la prueba Lambda de Wilks (Pérez, 2005, p. 328) la distancia entre los centroides de los grupos, siendo estos los promedios de las puntuaciones de cada función para las unidades de cada grupo.

Como resultado del análisis discriminante, se obtiene la matriz de coeficientes estandarizados de las funciones discriminantes canónicas que expresa la contribución de cada variable en la función discriminante, y la matriz de estructura que representa las correlaciones entre las funciones discriminantes y las variables. A partir de esta última se puede analizar qué variables son más influyentes en cada función discriminante, asignando cada variable a la función con la que tiene una mayor correlación absoluta.

Un desarrollo teórico y práctico de la técnica más detallado puede revisarse en Hair, Anderson, Tatham y Black (1999), Pérez (2009) o Torrado y Berlanga (2013), entre otros.

A continuación, y mediante esta técnica, se exploran las diferencias entre los cuatro grupos de municipios urbanos presentados en la sección anterior, y se determina cuáles de sus variables demográficas, sociales y económicas contribuyen más a las diferencias entre los grupos. Lamentablemente, la escasez de datos a nivel municipal ha limitado el número de variables utilizadas en el análisis a diez (Tabla 4).

La principal fuente de estadísticas municipales es el censo de población y viviendas que se realiza cada diez años. Los datos están disponibles en los censos de 2001 y 2011, aunque decidimos utilizar las variables correspondientes a 2001, ya que los valores de 2011 podrían verse afectados ya por las consecuencias de la crisis. Sin embargo, la correlación entre las variables de ambos censos es relativamente alta y los resultados son muy similares, independientemente del año considerado. La variable Densidad empresarial se tomó del Ministerio de Empleo y Seguridad Social de España y corresponde a 2007. A su vez, se utilizó el Índice turístico de 2004, calculado por el Anuario Económico de La Caixa de acuerdo con la recaudación impositiva de actividades turísticas. La Tabla 5 proporciona una visión general de 
los estadísticos de las variables utilizadas en el análisis y señala para qué variables las diferencias entre las medias de los grupos 1 y 4 (los que mejor y peor evolución tuvieron durante la crisis) son significativas, según el contraste U de Mann-Whitney (Pardo \& Ruiz, 2005).

\begin{tabular}{|c|c|c|}
\hline CATEGORÍA & VARIABLE & FUENTE \\
\hline \multirow{4}{*}{$\begin{array}{l}\text { Estructura } \\
\text { industrial }\end{array}$} & $\%$ trabajadores industria manufacturera & Instituto Nacional de Estadística (2001) \\
\hline & \% trabajadores construcción & Instituto Nacional de Estadística (2001) \\
\hline & $\%$ trabajadores actividades inmobiliarias & Instituto Nacional de Estadística (2001) \\
\hline & Índice turístico & La Caixa (2005) \\
\hline Diversidad & $\begin{array}{l}\text { Índice de Herfindalh de ocupación } \\
(\mathrm{CNAE}-1)\end{array}$ & $\begin{array}{l}\text { Basado en Instituto Nacional de } \\
\text { Estadística (2001) }\end{array}$ \\
\hline Capital humano & $\begin{array}{l}\% \text { población con estudios post-obliga- } \\
\text { torios }\end{array}$ & Instituto Nacional de Estadística (2001) \\
\hline Innovación & $\%$ trabajadores I+D & Instituto Nacional de Estadística (2001) \\
\hline Emprendimiento & $\begin{array}{l}\text { Densidad empresarial (Número de } \\
\text { empresas por } 1.000 \text { habitantes) }\end{array}$ & $\begin{array}{l}\text { Tesorería General de la Seguridad Socia } \\
(2007)\end{array}$ \\
\hline \multirow{2}{*}{$\begin{array}{l}\text { Factores } \\
\text { sociodemográficos }\end{array}$} & Densidad población & Instituto Nacional de Estadística (2001) \\
\hline & Edad media de población & Instituto Nacional de Estadística (2001) \\
\hline
\end{tabular}

TABLA 4 | Variables usadas en el análisis

FUENTE ELABORACIÓN PROPIA

Un primer análisis de los valores medios de los diferentes grupos permite observar que los municipios que tuvieron un peor comportamiento en estos años (Grupo 4) presentan, frente a los que mejor se comportaron (Grupo 1), menor densidad de población, menor nivel de estudios de su población, mayor tasa de trabajadores en la construcción y menor en actividades de I+D, mayor concentración de la ocupación y menor densidad empresarial.

El análisis discriminante revela que las variables difieren significativamente entre los grupos de resiliencia. Teniendo en cuenta que los municipios se han clasificado en cuatro grupos, el análisis discriminante define tres funciones en el modelo. La primera explica el $82,5 \%$ de la varianza, mientras que la segunda explica el 13,9\% y la tercera solo el 3,6\%. La prueba Lambda de Wilks muestra que las funciones 1 y 2 son significativas ( $p=0,000$ y 0,009 respectivamente), mientras que la tercera función no lo es $(p=0,466)$.

La Tabla 6 muestra la matriz de estructura. El coeficiente de correlación más alto entre cada variable y cualquier función discriminante se muestra en negrita e indica a qué función está más estrechamente ligada cada variable. Considerando esto, la función 1 podría identificarse como una Estructura industrial orientada a actividades con un valor agregado medio-alto; la función 2 como el (Relativamente bajo) grado de urbanización; y la función 3 como Diversidad de actividades. 


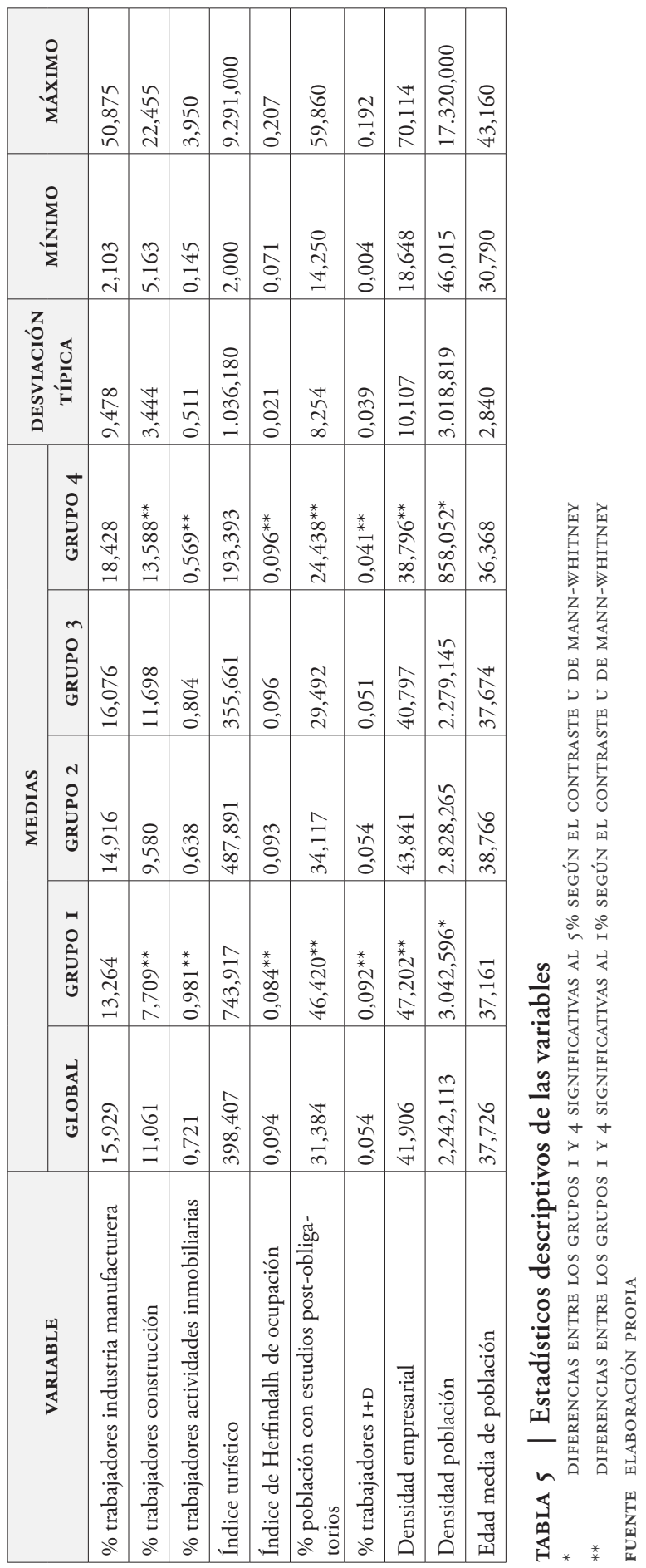




\begin{tabular}{|l|c|c|c|}
\hline \multicolumn{1}{|c|}{ VARIABLE } & FUNCIÓN I & FUNCIÓN 2 & FUNCIÓN 3 \\
\hline \% población con estudios pos-obligatorios & 0,819 & 0,007 & $-0,308$ \\
\hline \% trabajadores construcción & $-0,484$ & 0,378 & 0,279 \\
\hline \% trabajadores I+D & 0,280 & 0,199 & 0,057 \\
\hline Densidad empresarial & 0,207 & $-0,108$ & $-0,195$ \\
\hline \% trabajadores industria manufacturera & $-0,128$ & 0,104 & $-0,072$ \\
\hline Índice turístico & 0,123 & $-0,039$ & $-0,026$ \\
\hline Edad media de población & 0,120 & $-0,603$ & 0,056 \\
\hline Densidad de población & 0,181 & $-0,273$ & 0,225 \\
\hline \% trabajadores actividades inmobiliarias & 0,135 & 0,184 & 0,727 \\
\hline $\begin{array}{l}\text { Índice de Herfindalh de ocupación } \\
\text { (cNaE-1) }\end{array}$ & $-0,132$ & $-0,086$ & 0,203 \\
\hline Varianza explicada (\%) & 82,5 & 13,9 & 3,6 \\
\hline
\end{tabular}

TABLA 6 | Matriz de estructura

FUENTE ELABORACIÓN PROPIA

La Tabla 7 proporciona los centroides de cada uno de los grupos. Muestra que la función 1 diferencia principalmente entre los grupos 1 y 4, es decir, entre aquellos municipios en los que la crisis tuvo un menor impacto en el desempleo y aquellos donde el impacto fue mayor. La función 2 discrimina principalmente entre los grupos 1 y 2 , en otras palabras, entre municipios urbanos resilientes y relativamente resilientes.

\begin{tabular}{|l|c|c|c|c|}
\hline \multirow{2}{*}{ TIPOLOGÍAS } & \multirow{2}{*}{$\begin{array}{c}\text { NÚMERO DE } \\
\text { CASOS }\end{array}$} & \multicolumn{3}{|c|}{ FUNCIONES EN LOS CENTROIDES DE LOS GRUPOS } \\
\cline { 3 - 5 } & 12 & FUNCIÓN I & FUNCIÓN 2 & FUNCIÓN 3 \\
\hline Grupo 1 & 46 & 2,991 & 0,931 & $-0,073$ \\
\hline Grupo 2 & 59 & $-0,488$ & $-0,550$ & $-0,178$ \\
\hline Grupo 3 & 28 & $-1,502$ & 0,002 & 0,280 \\
\hline Grupo 4 & & 0,500 & $-0,266$ \\
\hline
\end{tabular}

TABLA 7 Funciones discriminantes y centroides de los grupos

FUENTE ELABORACIÓN PROPIA

Los municipios del Grupo 1, que muestran la mayor "resistencia" a la crisis, obtuvieron una puntuación muy alta en la función 1, en comparación con los otros grupos, que obtuvieron puntuaciones bajas e incluso negativas. Estos municipios tienen un mayor porcentaje de la población con estudios pos-obligatorios, una menor tasa de trabajadores empleados en la construcción y la industria y un mayor número de empleados en I+D, así como una mayor densidad empresarial y un mayor índice turístico que los municipios "menos resistentes". Los municipios del Grupo 1 también obtienen una puntuación alta en la función 2, mientras que los incluidos en el Grupo 2 tienen una puntuación negativa. Los municipios más resistentes a las crisis tienen una población más joven con patrones de asentamiento que son 
menos densos que los incluidos en el Grupo 2. Siguiendo a Burchell et al. (1998), Eurostat (2016) o Unión Europea (2011), estas dos variables podrían apuntar indirectamente a un grado de urbanización comparativamente más bajo, como el que se encuentra en los municipios suburbanos, en línea con los resultados obtenidos en investigaciones anteriores (Brakman et al., 2015).

Tanto la técnica utilizada como las circunstancias involucradas en su aplicación (solo 145 municipios), junto con la escasez de datos disponibles, limitan las conclusiones que pueden extraerse de estos resultados. A pesar de esto, parece confirmarse el vínculo positivo entre la resistencia del mercado laboral y los factores frecuentemente discutidos en el debate teórico, como el capital humano y la estructura industrial. Asimismo, aunque en menor grado, los resultados también confirman la relación negativa entre la resistencia y lo que podría entenderse como el grado de urbanización.

Un análisis de San Cugat del Vallés y Villarreal, dos municipios urbanos relativamente pequeńos (con menos de 100.000 habitantes) con tasas de desempleo similares en 2006, destaca la importancia de estos factores determinantes. En 2001, San Cugat del Vallés tenía un gran número de trabajadores empleados en actividades de I+D (80 trabajadores por 100.000 frente a 20 trabajadores por 100.000 en el caso de Villarreal) y un mayor porcentaje de la población con estudios pos-obligatorios (43,5\% en comparación con $20,4 \%$ en el caso del Villarreal). En consecuencia, San Cugat, un punto focal para la empresa privada que participa en proyectos innovadores destinados a consolidar el empleo, como el Triángulo de Innovación de Cataluña, y menos expuesto a los factores de riesgo, mostró una mayor resistencia al impacto de la crisis económica hasta 2013 (Bacaria, Borràs \& Fernández-Ribas, 2002; Pareja-Eastaway \& Pradel i Miquel, 2010). En contraste, Villarreal, dependiente en gran medida de la industria manufacturera y con un mayor número de trabajadores en el sector de la construcción, se vio más afectado por la crisis. Este impacto se sintió especialmente en el tejido empresarial, y particularmente en las pymes (Ruiz-Huerta, Benyakhlef \& Vizán, 2009). Esta tendencia se observó en toda la Comunidad Valenciana, una de las regiones españolas que perdió más empresas durante la crisis (Instituto Nacional de Estadística, 2017a).

San Sebastián y Castellón (con alrededor de 200.000 habitantes) proporcionan un ejemplo adicional que ilustra los patrones divergentes seguidos por los municipios urbanos de tamaño mediano, que evolucionaron de formas muy diferentes durante el periodo en cuestión. En 2006, el desempleo en Castellón fue menor que en San Sebastián, pero en 2013 fue siete puntos más alto. En 2001, el empleo en el municipio se centró en la industria manufacturera (más de un cuarto de la población activa en comparación con el 16,9\% del conjunto de municipios urbanos). Actividades como la producción de baldosas de cerámica, que se vio gravemente afectada por la dramática desaceleración en la construcción, hizo que el impacto de la crisis económica fuera particularmente grave en este municipio (Mañana, 2009). El municipio también se caracterizó por el número extremadamente bajo de trabajadores empleados en I+D y un porcentaje inferior a la media de población con estudios pos-obligatorios. En contraste, en 2001 San Sebastián destacó por el gran número de trabajadores empleados en I+D y el alto porcentaje de la población con 
estudios pos-obligatorios. Durante los noventa, el País Vasco adoptó una estrategia basada en clústeres y, más recientemente, una política centrada en la diversificación económica y la inversión en tecnología (Aranguren, Magro, Navarro \& Valdaliso, 2012). Este enfoque hacia los sectores con mayor valor agregado, junto con un impulso renovado para atraer el turismo, parece haber permitido a San Sebastián soportar mejor las consecuencias de la crisis.

Barcelona y Murcia, que superan los 400.000 habitantes, son dos ejemplos claros de diferente comportamiento frente a la crisis. En 2001, Murcia tenía un porcentaje menor de la población con estudios pos-obligatorios que Barcelona, un menor número de trabajadores empleados en $\mathrm{I}+\mathrm{D}$, una menor densidad empresarial y un mayor número de trabajadores del sector de la construcción. Murcia es un claro ejemplo del auge urbano de España, caracterizado por un aumento en la vivienda entre 2001 y 2008 que superó con creces el crecimiento de su población (García, 2010). Si bien su tasa de desempleo era más baja que la de Barcelona al inicio del periodo, se duplicó en comparación con la de Barcelona en el periodo 2006-2013.

\section{Conclusiones}

Este trabajo supone una aproximación parcial al concepto de resiliencia urbana en el corto plazo y pretende contribuir al análisis de la relación entre dicha condición y el grado de urbanización. El interés por la resiliencia en el ámbito urbano responde al papel fundamental que las ciudades podrían desempeñar en la resiliencia regional y por el conjunto de debilidades y fortalezas que las caracterizan y que actúan de modo opuesto sobre su capacidad de resistencia ante una crisis económica. Con el fin de contribuir a este debate, se analiza el impacto de la crisis en los mercados laborales de los grandes municipios urbanos españoles y se intenta caracterizar los municipios más y menos resistentes a partir de un conjunto de variables que con frecuencia se vinculan en la literatura a la resiliencia económica.

Los resultados del trabajo evidencian que, por lo general, los grandes municipios urbanos han sufrido con mayor intensidad los efectos de la actual crisis económica. Los municipios de más de 50.000 habitantes ya partían de tasas de desempleo más elevadas que los restantes municipios de sus respectivas regiones $\mathrm{y}$, como consecuencia de la crisis, estas diferencias se han visto incrementadas.

Por otra parte, los resultados del análisis discriminante parecen confirmar la relación positiva entre la resistencia del mercado de trabajo y factores como el capital humano y la innovación, al tiempo que se ha observado cierta relación negativa entre resiliencia a corto plazo y grado de urbanización. Tal vez por las características específicas de esta crisis, la estructura sectorial también ha resultado relevante (en particular a través del papel negativo del empleo en actividades como la construcción o las manufacturas tradicionales), aunque no así el grado de diversificación de dicha estructura. Los municipios con mayor cualificación de la fuerza de trabajo y empleo en innovación han mostrado mayores niveles de resistencia al impacto de la crisis en sus mercados de trabajo. De hecho, el nivel de desempeño de un municipio ha sido fundamentalmente condicionado por estos factores y no por otras cuestiones como su tamańo poblacional o la categoría funcional del área urbana en 
la que se incardina. Los resultados obtenidos permiten, por lo tanto, una serie de recomendaciones, en línea con las propuestas por Capello et al. (2015), apostando por ciudades que constituyan al mismo tiempo centro y fuente de actividades de alto valor añadido y alta calidad de los recursos.

Los resultados también muestran una brecha norte/sureste en la evolución de los mercados laborales locales en Espańa durante el periodo 2006-2013. Los grandes municipios urbanos que mejor han resistido la crisis se encuentran en las regiones del norte, mientras que las áreas urbanas más impactadas pertenecen a regiones del sur (Andalucía, Murcia, Comunidad Valenciana e Islas Canarias). Además, la crisis económica ha ampliado la brecha en términos de desigualdades espaciales, ya que ha empeorado la situación relativa de los grandes municipios urbanos situados en regiones que ya tenían tasas de desempleo elevadas y superiores a la media, con las modestas excepciones de Asturias y Galicia, y a pesar del empeoramiento relativo de los grandes municipios urbanos ubicados en la Comunidad Valenciana.

Existen limitaciones claras de nuestro estudio que deben ser señaladas. La primera está relacionada con la escasez de datos disponibles a nivel local, que imposibilita incluir variables que deben usarse en un análisis de esta naturaleza, y dificulta los análisis a largo plazo debido a la falta de continuidad en las series de datos. La falta de información estadística ha resultado en la imposibilidad de analizar, por ejemplo, los movimientos migratorios entre municipios interiores. Podría ser interesante — de cara a futuras investigaciones y en función de la disponibilidad de información-, analizar el impacto de la movilidad poblacional en la evolución de los mercados de trabajo urbanos. Una limitación adicional radica en que hemos asumido como mercado de trabajo local el marcado por los límites administrativos del municipio. Obviamente, somos conscientes de que esto dista de ser así, especialmente en los ámbitos urbanos (véase, por ejemplo, Lee, 2014). Por esa razón, en futuros trabajos se tratará de incluir, dentro del mercado de trabajo local urbano, el área de influencia real de los grandes municipios urbanos del país.

Igualmente, y en la medida en que el horizonte temporal lo permita, sería interesante complementar el análisis de la resistencia (2006-2013) con el de la capacidad de recuperación (2013 en adelante), obteniendo así un panorama más claro y completo del impacto real de la crisis de 2008 en los entornos urbanos de España.

\section{Referencias bibliográficas}

Adger, W. N. (2000). Social and ecological resilience: are they related? Progress in Human Geography, 24(3), 347-364. https://doi.org/10.1191/030913200701540465

Aranguren, M. J., Magro, E., Navarro, M. \& Valdaliso, J. M. (2012). Estrategias para la construcción de ventajas competitivas regionales. El caso del País Vasco. Madrid: Marcial Pons, Ediciones Jurídicas y Sociales.

Bacaria, J., Borràs, S. \& Fernandez-Ribas, A. (2002). Public action and innovation-support institutions in new technological agglomerations. European Urban and Regional Studies, 9(4), 283-296. https://doi.org/10.1177/096977640200900401 
Bailey, D. \& Chapain, C. (2011). The recession and beyond. Local and regional responses to the downturn. Abingdon: Routledge.

Brakman, S., Garretsen, H. \& van Marrewijk, C. (2015). Regional resilience across Europe: on urbanisation and the initial impact of the Great Recession. Cambridge Journal of Regions, Economy and Society, 8(2), 225-240. https://doi.org/10.1093/cjres/rsv005

Bristow, G. (2010). Resilient regions: re-'place'ing regional competitiveness. Cambridge Journal of Regions, Economy and Society, 3(1), 153-167. https://doi.org/10.1093/cjres/rsp030

Burchell, R. W., Shad, N. A., Listokin, D., Phillips, H., Sowns, A., Seskin, S., David, J. S., Moore, T., Helton, D. \& Gall, M. (1998). The costs of sprawl-Revisited. TCRP Report 39. Washington, DC: Transportation Research Board, National Research Council.

Capello, R., Caragliu, A. \& Fratesi, U. (2015). Spatial heterogeneity in the costs of the economic crisis in Europe: are cities sources of regional resilience? Journal of Economic Geography, 15(5), 951-972. https://doi.org/10.1093/jeg/lbu053

Caravaca, I., González-Romero, G. \& López, P. (2017). Crisis y empleo en las ciudades españolas. EURE, 43(128), 31-54. http://dx.doi.org/10.4067/S0250-71612017000100002

Champion, T. \& Townsend, A. (2011). The fluctuating record of economic regeneration in England's second-order city-regions, 1984-2007. Urban Studies, 48(8), 1539-1562. https://doi.org/10.1177/0042098010375320

Champion, T. \& Townsend, A. (2013). Great Britain's second-order city regions in recessions. Environment and Planning A: Economy and Space, 45(2), 362-382. https://doi. org/10.1068/a45100

Chapple, K. \& Lester, T. W. (2007). Emergingpatterns ofregional resilience. Working Paper, n. ${ }^{2} 2007-$ 13, Berkeley, CA: University of California, Institute of Urban and Regional Development (IURD). https://www.econstor.eu/bitstream/10419/59388/1/59285146X.pdf

Chapple, K. \& Lester, T. W. (2010). The resilient regional labour market? The US case. Cambridge Journal of Regions, Economy and Society, 3(1), 85-104. https://doi. org/10.1093/cjres/rsp031

Christopherson, S., Michie, J. \& Tyler, P. (2010). Regional resilience: theoretical and empirical perspectives. Cambridge Journal of Regions, Economy and Society, 3(1), 3-10. https:// doi.org/10.1093/cjres/rsq004

Clark, J., Huang, H.-I. \& Walsh, J. P. (2010). A typology of 'innovation districts': what it means for regional resilience. Cambridge Journal of Regions, Economy and Society, 3(1), 121-137. https://doi.org/10.1093/cjres/rsp034

Cohen, M. P. (2011). Cities in times of crisis: The response of local governments in light of the global economic crisis: The role of the formation of human capital, urban innovation and strategic planning. Working Paper, n. ${ }^{\circ}$ 2011-01. Berkeley, CA: University of California, Institute of Urban and Regional Development (IURD). https://www.econstor.eu/ bitstream/10419/59396/1/647147017.pdf

Comisión Europea (2009). Economic crisis in Europe: Causes, consequences and responses. European Economy \#7. Luxemburgo: European Commission. https://ec.europa.eu/ economy_finance/publications/pages/publication15887_en.pdf

Courvisanos, J., Jain, A. \& Mardaneh, K. K. (2016). Economic resilience of regions under crises: A study of the Australian economy. Regional Studies, 50(4), 629-643. https:// doi.org/10.1080/00343404.2015.1034669 
Cuadrado-Roura, J. R. \& Maroto, A. (2016). Unbalanced regional resilience to the economic crisis in Spain: a tale of specialisation and productivity. Cambridge Journal of Regions, Economy and Society, 9(1), 153-178. https://doi.org/10.1093/cjres/rsv034

Davies, S. (2011). Regional resilience in the 2008-2010 downturn: comparative evidence from European countries. Cambridge Journal of Regions, Economy and Society, 4(3), 369-382. https://doi.org/10.1093/cjres/rsr019

de la Dehesa, G. (2003). Balance de la economía espańola en los últimos veinticinco años. ice, Revista de Economía, 811, 53-79. http://www.revistasice.com/index.php/ICE/article/ view/570/570

Dijkstra, L., Garcilazo, E. \& McCann, P. (2015). The effects of the global financial crisis on European regions and cities. Journal of Economic Geography, 15(5), 935-949. https:// doi.org/10.1093/jeg/lbv032

Dubé, J. \& Polèse, M. (2016). Resilience revisited: Assessing the impact of the 2007-09 recession on 83 Canadian regions with accompanying thoughts on an elusive concept. Regional Studies, 50(4), 615-628. https://doi.org/10.1080/00343404.2015.1020291

Duranton, G. \& Puga, D. (2014). The growth of cities. En P. Aghion \& S. N. Durlauf (Eds.), Handbook of Economic Growth (pp. 781-853). Holanda Septentrional: Elsevier.

Eraydin, A. (2016). Attributes and characteristics of regional resilience: Defining and measuring the resilience of Turkish regions. Regional Studies, 50(4), 600-614. https://doi.org/10. 1080/00343404.2015.1034672

ESPON [European Spatial Planning Observation Network]. (2003). The role, specific situation and potentials of urban areas as nodes in a polycentric development. ESPON Project 1.1.1

- Third interim report. Luxembourg: EsPon. https://www.espon.eu/sites/default/files/ attachments/3.ir_1.1.1-full_0.pdf

Europa Publications (Ed.). (2009). Central and South-Eastern Europe 2009. Europa Regional Surveys of the World. London: Routledge.

Eurostat [Oficina Europea de Estadística] (Ed.). (2016). Urban Europe: Statistics on cities, towns and suburbs. 2016 Edition. Luxemburgo: Publications office of the European Union. https://doi.org/10.2785/91120

Eurostat [Oficina Europea de Estadística]. (2018). Eurostat Database. European Commission. https://ec.europa.eu/eurostat/data/database

Fisher, R. A. (1936). The use of multiple measurements in taxonomic problems. Annals of Eugenics, 7(2), 179-188. https://doi.org/10.1111/j.1469-1809.1936.tb02137.x

Fotheringham, A. S. \& Wong, D. W. S. (1991). The modifiable areal unit problem in multivariate statistical analysis. Environment and Planning A: Economy and Space, 23(7), 1025-1044. https://doi.org/10.1068/a231025

Fratesi, U. \& Perucca, G. (2018). Territorial capital and the resilience of European regions. The Annals of Regional Science, 60(2), 241-264. https://doi.org/10.1007/s00168-0170828-3

García, M. (2010). The breakdown of the Spanish urban growth model: social and territorial effects of the global crisis. International Journal of Urban and Regional Research, 34(4), 967-980. https://doi.org/10.1111/j.1468-2427.2010.01015.x

Giannakis, E. \& Bruggeman, A. (2017). Economic crisis and regional resilience: evidence from Greece. Papers in Regional Science, 96(3), 451-476. https://doi.org/10.1111/ pirs. 12206 
Glaeser, E. \& Kahn, M. (2004). Sprawl and urban growth. En J. V. Henderson \& J.-F. Thisse (Eds.), Handbook of Regional and Urban Economics (pp. 2481-2527). Holanda Septentrional: Elsevier.

Hair Jr., J. F., Anderson, R. E., Tatham, R. L. \& Black, W. C. (1999). Análisis Multivariante (5a ed.). Madrid: Prentice Hall Iberia.

Han, Y. \& Goetz, S. J. (2015). The economic resilience of US counties during the great recession. The Review of Regional Studies, 45(2), 131-149. https://rrs.scholasticahq. com/article/8059-the-economic-resilience-of-u-s-counties-during-the-great-recession

Harvey, D. (2012). Las raíces urbanas de las crisis financieras. En J. Bell, M. Borja \& M. Corti (Eds.), Ciudades, una ecuación imposible (pp. 321-358). Barcelona: Icaria.

Hill, E., St. Clair, T., Wial, H., Wolman, H., Atkins, P., Blumenthal, P., Ficenec, S. \& Friedhoff, A. (2012). Economic shocks and regional economic resilience. En M. Weir, N. Pindus, H. Wial \& H. Wolman (Eds.), Urban and Regional Policy and Its Effects: Building Resilient Regions (pp. 193-274). Washington, DC: Brookings Institution Press.

Hill, E., Wial, H. \& Wolman, H. (2008). Exploring regional economic resilience, Working Paper - n. ${ }^{\circ}$ 2008-04. Berkeley, CA: University of California, Institute of Urban and Regional Development (IURD). https://www.econstor.eu/ bitstream/10419/59420/1/592859940.pdf

Holling, C. S. (1973). Resilience and stability of ecological systems. Annual Review of Ecology and Systematics, 4, 1-23. https://doi.org/10.1146/annurev.es.04.110173.000245

Hudson, R. (2010). Resilient regions in an uncertain world: wishful thinking or a practical reality? Cambridge Journal of Regions, Economy and Society, 3(1), 11-25. https://doi. org/10.1093/cjres/rsp026

Instituto Nacional de Estadística (2001). Censo de Población y Viviendas 2001. Madrid: Instituto Nacional de Estadística. http://ine.es/censos2011_datos/cen11_datos_resultados.htm

Instituto Nacional de Estadística (2012). Cifras oficiales de población de los municipios españoles: Revisión del Padrón Municipal. Madrid: Instituto Nacional de Estadística. http://ine. es/dyngs/INEbase/es/operacion.htm?c=Estadistica_C\&cid=1254736177011\&menu $=$ resultados\&idp $=1254734710990$

Instituto Nacional de Estadística (2017a). Directorio Central de Empresas. Madrid: Instituto Nacional de Estadística. http://www.ine.es/dyngs/INEbase/es/operacion.htm?c=Estad istica_C\&cid $=1254736160707 \&$ menu=ultiDatos\&idp $=1254735576550$

Instituto Nacional de Estadística (2017b). Encuesta de Población Activa. Madrid: Instituto Nacional de Estadística. http://www.ine.es/dyngs/INEbase/es/operacion.htm?c=Estad istica_C\&cid $=1254736176918 \&$ menu $=$ ultiDatos\&idp $=1254735976595$

La Caixa (2005). Anuario Económico de España 2004. Barcelona: Servicio de Estudios de La Caixa.

La Caixa (2013). Anuario Económico de España 2012. Barcelona: Servicio de Estudios de La Caixa.

Lee, N. (2014). Grim down South? The determinants of unemployment increases in British cities in the 2008-2009 recession. Regional Studies, 48(11), 1761-1778. https://doi.or g/10.1080/00343404.2012.709609

Lee, N., Morris, K. \& Jones, A. (2009). Recession and recovery: How UK cities can respond and drive the recovery. Londres: The Work Foundation. 
Maddison, A. (2009). Statistics on World Population, gdp and Per Capita GDP, 1-2008 AD. Groningen: University of Groningen, Groningen Growth \& Development Centre. http://www.ggdc.net/maddison/oriindex.htm

Mañana, R. (2009). Un ensayo en prospectiva: la industria minera en el futuro de la economía española. Mediterráneo Económico, 16, 129-153.

Markusen, A. (1999). Fuzzy concepts, scanty evidence, policy distance: the case for rigour and policy relevance in critical regional studies. Regional Studies, 33(9), 869-884. https:// doi.org/10.1080/00343400701232348

Martin, R. (2012). Regional economic resilience, hysteresis and recessionary shocks. Journal of Economic Geography, 12(1), 1-32. https://doi.org/10.1093/jeg/lbr019

Martin, R., Sunley, P., Gardiner, B. \& Tyler, P. (2016). How regions react to recessions: Resilience and the role of economic structure. Regional Studies, 50(4), 561-585. https://doi.org/10.1080/00343404.2015.1136410

McCann, P. (2013) Modern urban and regional economics. Oxford: Oxford University Press.

Méndez, R., Abad, L. D. \& Echaves, C. (2015). Atlas de la crisis. Valencia: Tirant lo Blanch.

Mrinska, O. \& Smetkowski, M. (2013). Report on the second seminar of the RSA research network on the impact of the global economic crisis on capital cities. Regions, 290(1), 29-31. https://doi.org/10.1080/13673882.2013.10715708

Muñoz de Bustillo, R. (2007). Spain: The paradox of job insecurity alongside high employment growth. En F. Eyraud \& D. Vaughan-Whitehead (Eds.), The Evolving World of Work in the Enlarged EU: Progress and Vulnerability (pp. 439-480). Ginebra: International Labour Office.

Muñoz de Bustillo, R. \& Antón, J. I. (2010). From sending to host societies: immigration in Greece, Ireland and Spain in the 21st century. Industrial Relations Journal, 41(6), 563583. https://doi.org/10.1111/j.1468-2338.2010.00586.x

Openshaw S. (1984). The modifiable areal unit problem. Norwich: GeoBooks.

Palaskas, T., Psycharis, Y., Rovolis, A. \& Stoforos, C. (2015). The asymmetrical impact of the economic crisis on unemployment and welfare in Greek urban economies. Journal of Economic Geography, 15(5), 973-1007. https://doi.org/10.1093/jeg/lbv027

Pardo, A. \& Ruiz, M. A. (2005). Análisis de datos con spss 13 Base. Madrid: McGraw-Hill.

Pareja-Eastaway, M. \& Pradel i Miquel, M. (2010). New economy, new governance approaches? Fostering creativity and knowledge in the Barcelona Metropolitan Region. Creative Industries Journal, 3, 29-46. https://doi.org/10.1386/cij.3.1.29_1

Parkinson, M., Meegan, R., Karecha J., Evans, R., Jones, G., Tosics, I. \& Hall, P. (2012) Second tier cities in Europe: In an age of austerity why invest beyond the capitals? Liverpool: ESPON \& Institute of Urban Affairs, Liverpool John Moores University. https://people. uta.fi/ atmaso/verkkokirjasto/Second_tier_cities_policy.pdf

Pendall, R., Foster, K. A. \& Cowell, M. (2007). Resilience and regions: Building understanding of the metaphor. Working Paper No. 2007-12. Berkeley, CA: University of California, Institute of Urban and Regional Development (IURD). https://iurd.berkeley.edu/ wp/2007-12.pdf

Pendall, R., Foster, K. A. \& Cowell, M. (2010). Resilience and regions: Building understanding of the metaphor. Cambridge Journal of Regions, Economy and Society, 3(1), 71-84. https://doi.org/10.1093/cjres/rsp028

Pérez, C. (2005). Métodos estadísticos avanzados con SPSS. Madrid: Thomson Editores Spain. 
Pérez, C. (2009). Técnicas estadísticas multivariantes con SPSS. Madrid: Ibergaceta Publicaciones, S.L.

Pike, A., Dawley, S. \& Tomaney, J. (2010). Resilience, adaptation and adaptability. Cambridge Journal of Regions, Economy and Society, 3(1), 59-70. https:/doi.org/10.1093/cjres/ rsq001

Polèse, M. (2010). The resilient city: on the determinants of successful urban economies. Working Paper n. ${ }^{\circ}$ 2010-03. Quebec: University of Quebec, Centre - Urbanisation Culture Société. https://pdfs.semanticscholar.org/669d/805385f043c6c60e7c3b7f10277ec1def7fb. pdf

Ruiz-Huerta, J., Benyakhlef, M. \& Vizán, C. (2009). Las Comunidades Autónomas ante la crisis económica: impacto territorial de la recesión, políticas autonómicas de reactivación y tensiones en las cuentas públicas. Informe Comunidades Autónomas, 2009, 89-112. http://idpbarcelona.net/docs/public/iccaa/2009/informe_2009.pdf

Sacht, S. (2015). On the efficiency of labor market reforms: how to solve the Spanish puzzle? Economics: The Open-Access, Open-Assessment E-Journal, 9(44), 1-30. https://doi. org/10.5018/economics-ejournal.ja.2015-44

Shaw, K. (2012). The rise of the resilient local authority? Local Government Studies, 38(3), 281300. https://doi.org/10.1080/03003930.2011.642869

Solé-Ollé, A. \& Viladecans-Marsal, E. (2011). Local spending and the housing boom. Cities and innovation. Document de treball de l'IEB 2011/27. Barcelona: Universitat de Barcelona, Institut d'Economia de Barcelona. http://diposit.ub.edu/dspace/ bitstream/2445/116576/1/IEB11-27_SoleOlle.pdf

Tesorería General de la Seguridad Social (2007). Estadísticas TGSS. Madrid: Ministerio de Empleo y Seguridad Social. https://w6.seg-social.es/PXWeb/pxweb/es/Estad\%c3\%adsticas\%20 TGSS/

Torrado, M. \& Berlanga, V. (2013). Análisis discriminante mediante spss. Revista d'Innovació $i$ Recerca en Educació (REIRE), 6(2), 150-166. https://doi.org/10.1344/reire2013.6.26210

Townsend, A. \& Champion, T. (2014). The impact of recession on city regions: The British experience, 2008-2013. Local Economy: The Journal of the Local Economy Policy Unit, 29(1-2), 38-51. https://doi.org/10.1177/0269094213518885

Unión Europea (2011). Ciudades del mañana. Retos, visiones y caminos a seguir. Bruselas: Unión Europea.

URBACT (2010). Cities and the economic crisis. A survey on the impact of the economic crisis and the responses of URBACT II cities. https://urbact.eu/sites/default/files/import/general_library/ Survey_CitiesandCrisis_01.pdf

van Ark, B., O’Mahony, M. \& Ypma, G. (2007). The eu KLems productivity report. An Overview of Results from the EU KLEMS Growth and Productivity Accounts for the European Union, eu Member States and Major Other Countries in the World. N.o 1. Groningen: University of Groningen, Groningen Growth and Development Centre. http://www. euklems.net/data/eu_klems_productivity_report1.pdf

Viñuela, A., Rubiera, F. \& Cueto, B. (2012). Espacio y empleabilidad. ¿Importa el concepto de región? ICE, Revista de Economía, 865, 155-168. http://www.revistasice.com/index. $\mathrm{php/ICE/article/view/1500/1500}$ 